\title{
Study of Hydrate Formation and Inhibition for LPG
}

\author{
Mridul Sarker, Debasish Chowdhury, Iqbal Hossain* \\ Department of Chemical Engineering, Bangladesh University of Engineering \& Technology, \\ BUET, Dhaka-1000.
}

\begin{abstract}
Liquefied petroleum gas (LPG) has already been proved as an efficient fuel and feedstock for many chemical and petrochemical industries. Hydrate formation can create serious problems in the production, transmission, storage, and end-use of LPG. Hence an adequate knowledge on hydrate formation (i.e., the effects of moisture content and pressure on the hydrate formation temperature) is crucial to maintain the process conditions out of the hydrate formation region. However the study particularly on such aspects is scarcely available in literature. Therefore the effects of moisture content and pressure on the LPG hydrate formation temperature are studied extensively. A correlation is also developed to predict hydrate formation temperature for various LPG compositions. Hydrate inhibition performances of various inhibitors are also evaluated. Aspen-HYSYS process simulator is used to perform the study. The present study provides useful information to reduce the hydrate formation of LPG.
\end{abstract}

\section{INTRODUCTION}

Liquefied petroleum gas, also called LPG, is a flammable mixture of hydrocarbon gases, mostly propane and butane, along with trace amounts of higher hydrocarbons. It is a clean-burning and environmental friendly efficient fuel with minimum sulphur content, which can be a vital source of energy for next generation low carbon world due to an immediate and global availability, its natural by-product origin, transportation flexibility and diverse applications. Millions of people presently use LPG and depend on it for thousands of applications, in commercial business, in industry, transportation, farming, power generation, cooking, and heating purposes. LPG hydrates are solids crystalline compounds, resembling ice or wet snow in appearance, that form from a combination of moisture and one or more hydrocarbon gases. Formation of gas hydrates depends on several criterion and they are stable only under specific pressure-temperature conditions. Gas hydrates can cause several problems in the production, transportation, storage and use of liquefied petroleum gases. Hydrate formation can be prevented by injecting additives as hydrate inhibitor. Injection of inhibitor also depends on compositions of LPG and degree of inhibition required. Appropriate and specific data on LPG hydrate formation conditions and prevention of LPG hydrate formation by inhibitors are important to know which are hardly available in literature. Particularly the non-ideality of LPG mixture due to the presence of moisture in commercial LPG must be accounted in the prediction of hydrate formation conditions. Therefore, the hydrate formation conditions and inhibition of hydrates of LPG is studied in the present research considering the non-ideality (i.e., presence of moisture). To carry out this study the Aspen Hysys V7.3 process simulator is employed.

\section{METHODOLOGY}

Pure experimental setup with high accuracy and consistency for evaluating hydrate formation temperature is rarely available. Hence, simulation approach is adopted for estimating hydrate formation temperature. Aspen HYSYS process simulator is a core element of AspenTech's aspenONE® Engineering applications ${ }^{[1]}$. It has already been proved as an efficient simulator with reasonable accuracy. It offers a comprehensive thermodynamics foundation for accurate determination of physical properties, transport properties, and phase behavior. Aspen HYSYS can be used to determine outlet process conditions if the inlet conditions like temperature, pressure, and composition are specified. Therefore, a LPG mixture or stream is simulated combining propane, n-butane, and other components of desired compositions at the specified condition in user interface of Aspen-HYSYS as shown in fig. 1. Hydrate formation utility tool of Aspen-Hysys can generally be used to determine hydrate formation temperature. The hydrate formation utility calculates the incipient solid formation point for hydrates in a given stream. The predictive models are based on fundamental thermodynamic principles and use equation-of-state generated properties in calculating the equilibrium conditions. These predictive models can therefore be applied to various compositions and extreme operating conditions with a greater degree of reliability than one may expect with empirical expressions or charts. Hence, this utility tool is used to carry out this study, for the simulated LPG stream as shown in Fig. 2. CSM model, a new hydrate model developed by the Colorado School of Mines (CSM) is used to find out hydrate formation temperature in this study. This model also follows the formulation of Van Der Waals and Platteeuw but with very significant enhancements to thermodynamic models for the hydrate and aqueous phases [1]. CSM model can be used for all possible scenarios; vapor- 
hydrate, liquid-hydrate, aqueous-hydrate, vapor-liquidhydrate, vapor-aqueous-hydrate, and vapor-liquidaqueous-hydrate (where liquid refers to a hydrocarbon liquid). This model is used for hydrate predictions of the types I and II and for cases with and without inhibitors. The CSM model can be used with the SRK, Peng-Robinson and Glycol property packages. In this current study Peng-Robinson is chosen as fluid package to complete the simulation of LPG stream.

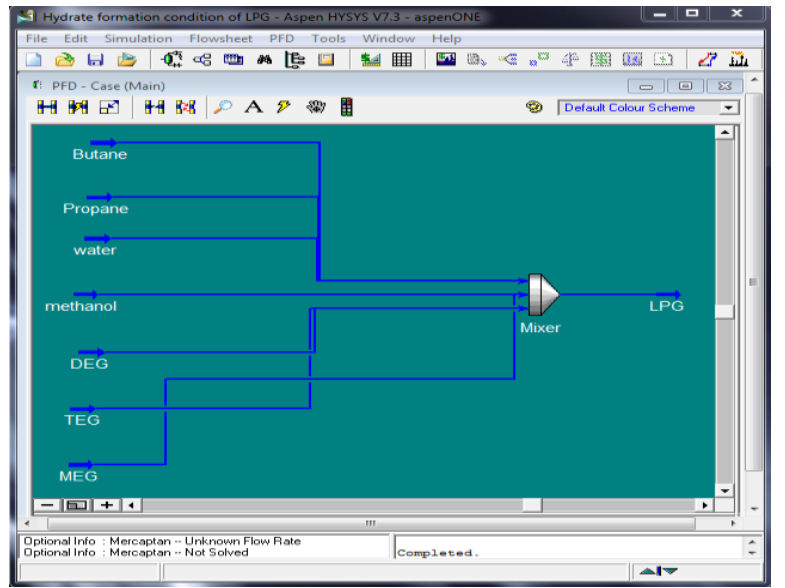

Fig. 1: Simulation of LPG mixture for evaluating hydrate formation temperature by Aspen-Hysys

Commercial LPG always contains different impurities like moisture, methyle-mercaptan, higher and lower hydrocarbons. Nevertheless, in this study only moisture content is considered as impurity to avoid complexity and to investigate the effect of free water content on hydrate formation temperature.

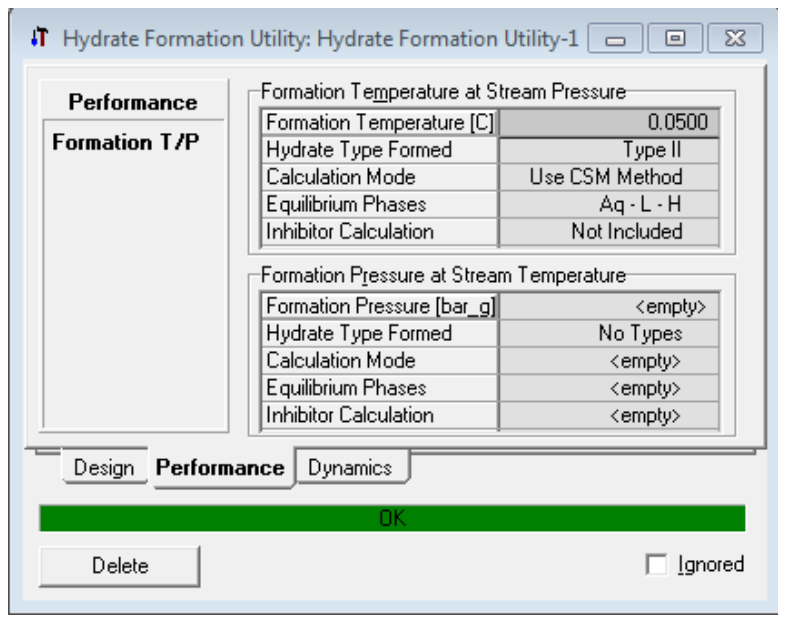

Fig. 2: Hydrate Formation Utility

The analysis is done by plotting several curves at different operating pressures and moisture contents to figure out the dependency of hydrate formation temperature on these factors. In this study the range of moisture content used is $2.5-10 \%$ of total LPG mixture and range of pressure used is 5 - 20 bar as these pressure range is very common for LPG handling i.e. storage, transporting, and bottling etc ${ }^{[2]}$. Hysys process simulation is done to explore effect of inhibition of different inhibitors (e.g., methanol, ethylene glycol, diethylene glycol, and triethylene glycol). A curve is also produced to find out inhibitor amount needed to inhibit hydrate up to a desired degree of inhibition.

\section{RESULTS AND DISCUSSION}

LPG hydrates are included in a general class of compound known as clathrates, which have a structure wherein guest molecules are entrapped in a cage-like framework of host molecules without forming a chemical bond. Two types of crystalline structure have been proposed for hydrates. Smaller molecules, such as methane, ethane and hydrogen sulfide form a body centered cubic lattice called structure I. Structure II is a diamond lattice such as propane and isobutane. LPG gas mixture form structure II type hydrates ${ }^{[3]}$. The maximum temperature at which LPG hydrate can form depends on pressure and gas composition. Below freezing point of water hydrate of LPG can coexist with ice from water. But under the appropriate pressure, they can exist at temperatures significantly above the freezing point of water.

\subsection{Effect of Moisture Content on Hydrate Formation Temperature}

An addition of very small amount of moisture in propane-butane mixture results in a sudden reduction of hydrate formation temperature from initial point. Several curves are plotted for a reasonable moisture content range $(2.5 \%, 5 \%$, and $7.5 \%)$ at certain pressure to determine the effect of further addition of moisture content on hydrate formation temperature.

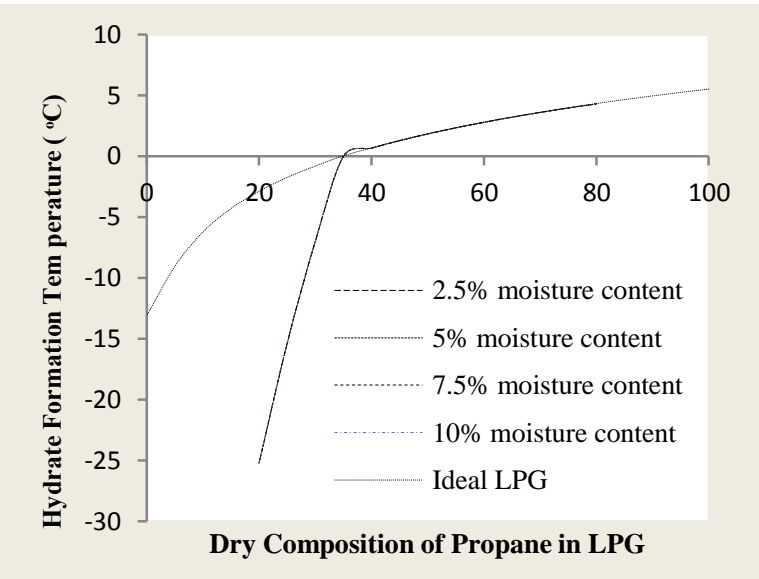

Fig. 3(a): Hydrate Formation Temperature Vs Dry

Composition of Propane for different moisture content in commercial LPG at 5 bar pressure

Fig. 3(a) shows almost same trend of curve drawn for different moisture content at constant pressure. For propane composition lower than 35\%, hydrate formation temperature is below freezing point of water and it rises quite abruptly with composition of propane in LPG that means slope is very steep in this region. 
And for propane composition higher than $35 \%$, hydrate formation temperature exceeds freezing point of water but the rate of change of hydrate formation temperature with propane composition is quite small with respect to the rate of change below freezing point region. Similar figures are plotted for different pressures (i.e., LPG bottling pressure 10 bar, LPG cylinder pressure 20 bar) to observe the trend of curves. These curves also represent the findings of fig.3 (a).

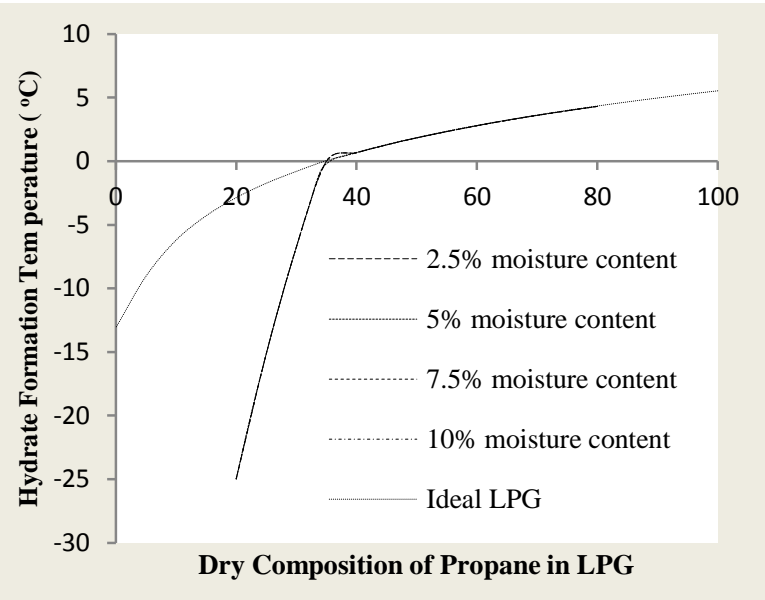

Fig. 3(b): Hydrate Formation Temperature Vs Dry Composition of Propane for different moisture content in commercial LPG at 10 bar pressure

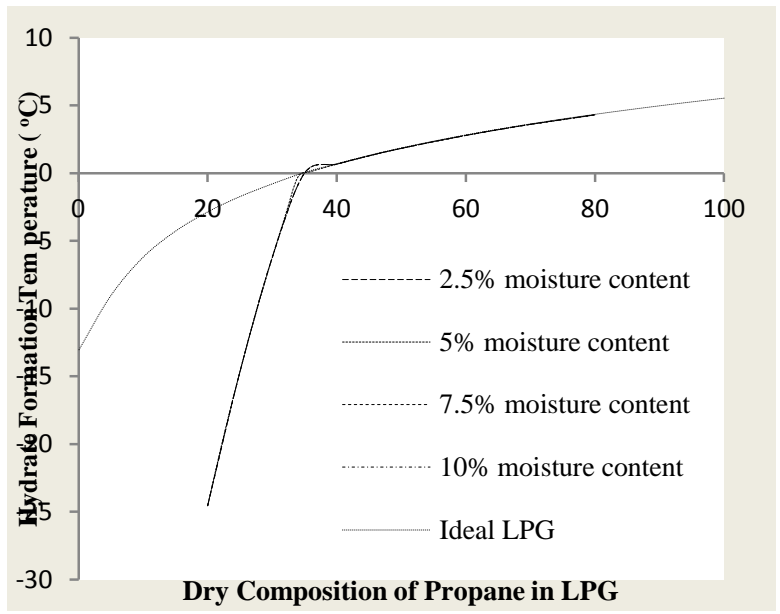

Fig. 3(c): Hydrate Formation Temperature Vs Dry Composition of Propane for different moisture content in commercial LPG at 20 bar pressure

\subsection{Effect of Operating Pressure on Hydrate Formation Temperature}

Operating pressure is another key factor for hydrate formation temperature. To investigate the effect of changing pressure few curves are plotted at different pressure i.e., LPG storage pressure 5 bar, LPG bottling pressure 10 bar, LPG cylinder pressure 20 bar for a constant $2.5 \%$ moisture content. Curves are shown below in fig. 4(a)

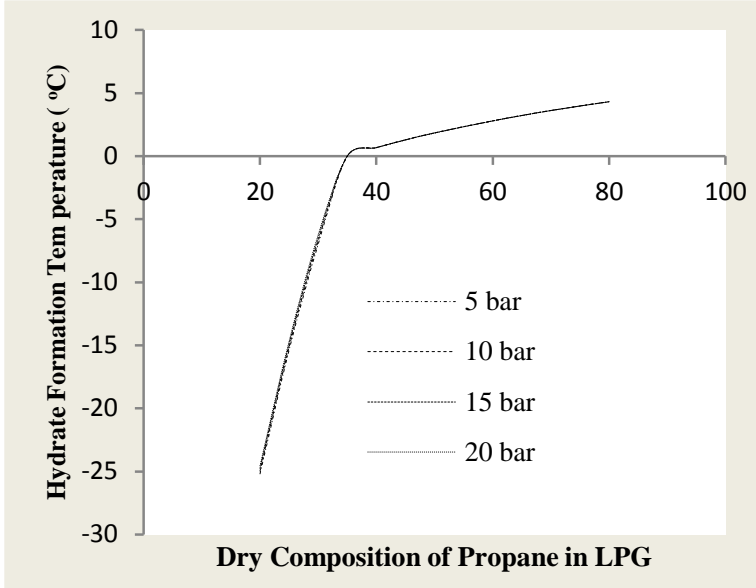

Fig. 4(a): Hydrate Formation Temperature Vs Dry Composition of Propane for different pressure in commercial LPG with $2.5 \%$ moisture content

Fig. 4(a) exhibits similar trend for different operating pressure at constant moisture content. For a constant composition of LPG, hydrate formation temperature increases slowly with the increase of operating pressure. Thus pressure has a definite but minor contribution to hydrate formation temperature. There is no significant change in graphical trend due to pressure. It has similarity with previous curve in fig. 3(a) for composition of propane lower and higher than $35 \%$; in which hydrate formation temperature is coincide with freezing point of water. Similar curves are plotted for different moisture content (5\% and $10 \%)$ to observe the trend of curves. These curves also represent the findings of fig.4 (a).

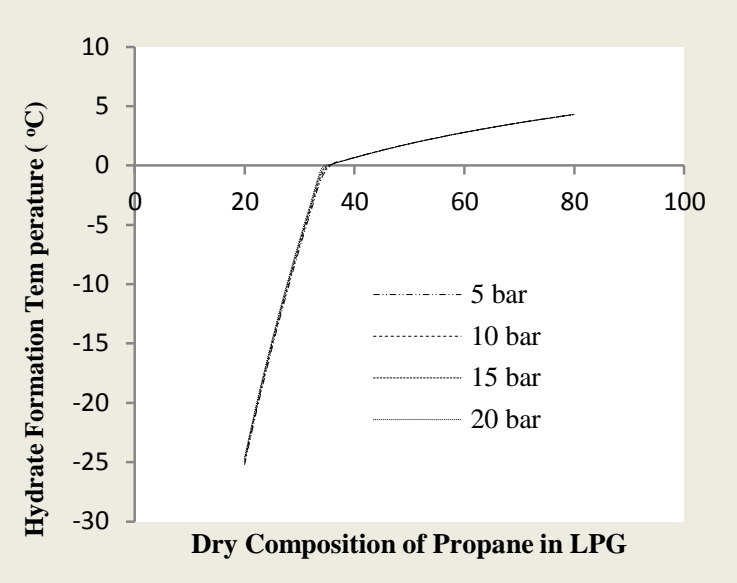

Fig. 4(b): Hydrate Formation Temperature Vs Dry Composition of Propane for different pressure in commercial LPG with 5\% moisture content 


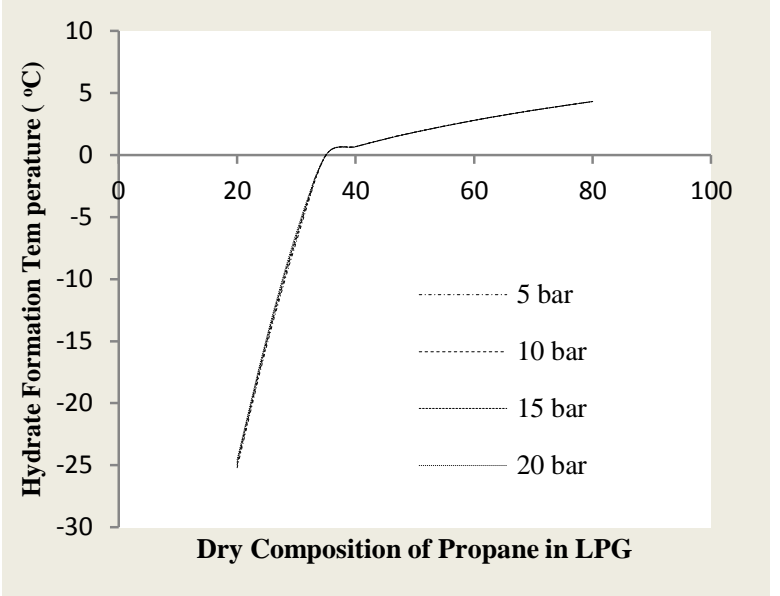

Fig. 4(c): Hydrate Formation Temperature Vs Dry Composition of Propane for different pressure in commercial LPG with $7.5 \%$ moisture content

Studying all these curves, it is very obvious that the dependency of hydrate formation temperature on moisture content is negligible and on operating pressure is very little. Hydrate formation temperature is proportional to operating pressure and composition of propane in LPG which is the main key factor for change of hydrate formation temperature.

\subsection{A Correlation for Prediction of Hydrate Formation Temperature}

There are several theoretical and empirical correlations in literature which can quite accurately predict hydrate formation temperature for certain pressure and composition. For general purposes like bottling, shipping and transportation, low pressure range like from 5 to 20 bar is required for dry composition of propane ranging from 20 to $80 \%$. From following graphs it is clear that hydrate formation temperature is quite independent of pressure and moisture content and varies greatly with propane composition. Two correlations are developed in order to calculate hydrate formation temperature.

For propane composition below $35 \%$,

Hydrate formation temperature,

$\mathrm{T}\left({ }^{\circ} \mathrm{C}\right)=183.4 \mathrm{X}-59.68$

Where, $\mathrm{X}$ is composition of propane in LPG mixture

For propane composition above $35 \%$,

Hydrate formation temperature,

$\mathrm{T}\left({ }^{\circ} \mathrm{C}\right)=9.969 \mathrm{X}-3.062$

Where, $\mathrm{X}$ is composition of propane in LPG mixture

In these correlations effect of pressure and moisture content is neglected. But such exclusion introduces error $3 \%$ to $5 \%$ which can be take into consideration.

\subsection{Effect of Inhibition on Hydrate Formation Temperature}

Additives injection is generally required for LPG in order to prevent corrosion and hydrate formation in the storage and transmission system. The most common additives are methanol, Ethylene glycol (MEG), Diethylene glycol (DEG), Triethylene glycol (TEG) ${ }^{[3]}$. Fig. 5(a) presents the comparison of inhibition effect of Methanol, Ethylene glycol (MEG), Diethylene glycol (DEG), Triethylene glycol (TEG) and 1:1 mixture of Methanol and Ethylene glycol as inhibitor at constant operating pressure and constant moisture content of commercial LPG.

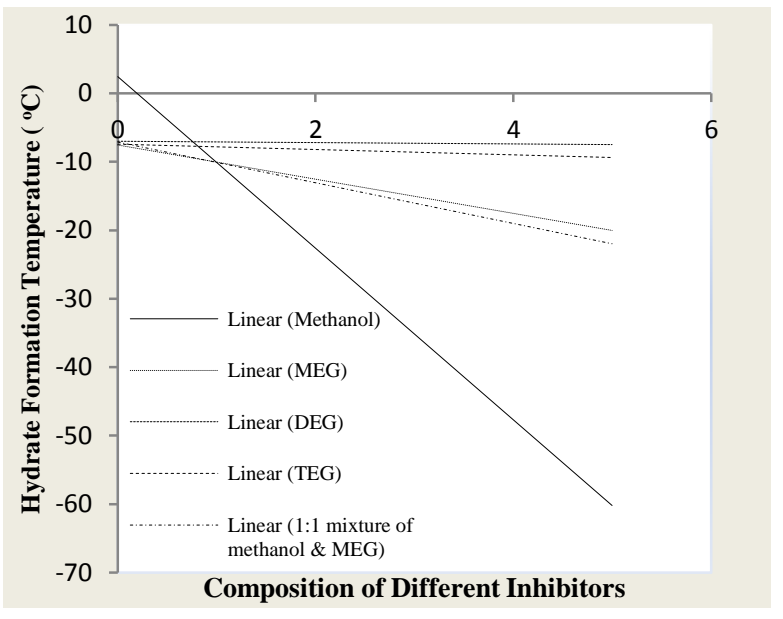

Fig. 5(a): Hydrate Formation Temperature vs. Composition of Different Inhibitors

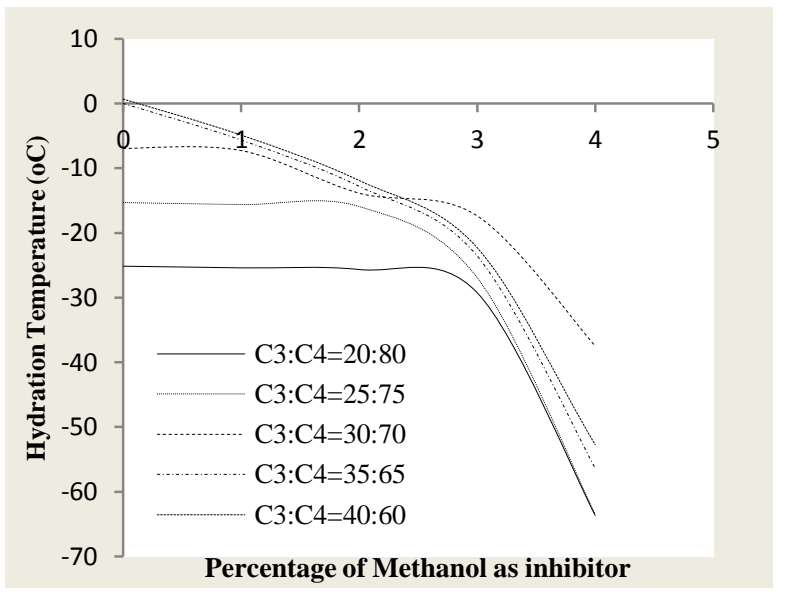

Fig. 5(b): Hydrate Formation Temperature Vs Composition of methanol as inhibitor in commercial LPG with 5\% moisture content at 5 bar pressure 
This figure shows that the degree of inhibition is very high when methanol is used as inhibitor. It can reduce hydrate formation temperature up to $-60^{\circ} \mathrm{C}$ or completely prohibit hydrate formation only by injecting 1 to 5 percent of total LPG mixture. High vapor pressure of methanol, the lowest molecular weight alcohol, makes it a very effective mean to eliminate hydrate formation as it disperses well in gas stream. Readily availability in bulk and the least expensiveness makes methanol the most attractive inhibitor among all. Moreover methanol added heating value of fuel because it does not require recovery.

Degree of inhibition varies with the change of compositions of commercial LPG and also requirement of degree of inhibition is different for different working condition and environment. A graph is plotted to determine the requirement of methanol as inhibitor to reduce hydrate formation temperature to a certain range for different composition of commercial LPG with certain moisture content at constant pressure. As hydrate formation temperature is not very much dependent on pressure and moisture content, this plot can be proved very efficient to find out amount of inhibitor needed to carry out inhibition up to a degree desired according to operating environment and condition. This graph is shown below in fig. 5(b).

\section{CONCLUSIONS}

Commercial LPG always contains an amount of free moisture. This moisture can be present in LPG originally as an impurity and can also be extracted from cargo tank bulkheads if rust is present. Propane and butane, core element of LPG, form hydrates under certain conditions of temperature and pressure in the presence of free moisture. Among the key circumstances that are essential for hydrate formation, the effect of composition of hydrocarbons, moisture content, operating pressure are thoroughly analyzed in this study. The effect of free moisture content on hydrate formation temperature is negligible and the effect of operating pressure is very diminutive. Thus hydrate formation temperature is greatly a function of the composition of propane in LPG mixture. The higher the composition of propane the higher is the hydrate formation temperature. The correlation proposed predicts hydrate formation temperature at various composition of propane reasonably. Among other inhibitors methanol is the best one to inhibit hydrate formation. The addition of 1 to $5 \%$ methanol is required to inhibit hydrate formation based on the degree of inhibition required.

\section{REFERENCES}

[1] HYSYS. Aspen HYSYS user guide. Aspen Technology Inc. www.aspentech.com

[2] Basundhara LP gas Limited, 2010, product, viewed 05 August 2012, <http://www.bashundharagroup.com/LPGas/ind ex.php?view=products $>$.

[3] Kumar Sanjay, Gas Production Engineering, Gulf Publishing Company, Texas, 1978, p. 184,208 .

[4] Sharareh, Ameripour 2005, 'Prediction of GasHydrate Formation Conditions In Production and Surface Facilities', MSc thesis, Texas A\&M University, Texas, USA.

[5] Scribd, 2012, viewed 6 August 2012, <http://www.scribd.com/doc/94952383/Docs $>$.

[6] Scribd, 2012, viewed 6 August 2012, <http://www.scribd.com/doc/44640867/Hydrates>.

[7] Sloan, E.D. Jr.: Clathrate Hydrates of Natural Gases, Marcel Dekker, New York (1990).

[8] E Berecz,M.Balla-Achs, Gas Hydrates, Elsevier Science Publishing Company,Inc, New York, 1983. 